\title{
On the number of representations providing noiseless subsystems
}

\author{
William Gordon Ritter \\ Harvard University, Department of Physics
}

(Dated: July 24, 2005)

\begin{abstract}
This paper studies the combinatoric structure of the set of all representations, up to equivalence, of a finite-dimensional semisimple Lie algebra. This has intrinsic interest as a previously unsolved problem in representation theory, and also has applications to the understanding of quantum decoherence. We prove that for Hilbert spaces of sufficiently high dimension, decoherence-free subspaces exist for almost all representations of the error algebra. For decoherence-free subsystems, we plot the function $f_{d}(n)$ which is the fraction of all $d$-dimensional quantum systems which preserve $n$ bits of information through DF subsystems, and note that this function fits an inverse beta distribution. The mathematical tools which arise include techniques from classical number theory.

PACS numbers: 03.65.Yz, 03.67.Pp, 03.65.Fd, 02.20.Qs
\end{abstract}




\section{INTRODUCTION}

A variety of schemes for protecting quantum information have been developed, including

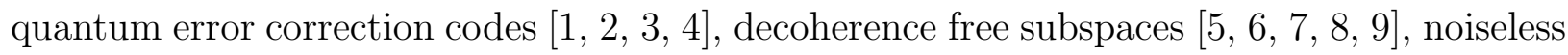
subsystems [10], bang-bang decoupling [11], and topological quantum computation [12]. The first four of these techniques are closely related to each other and can be described in a simple unified framework based on representations of the algebra of errors [10, 13, 14]. More recently, it was shown [15] that topological quantum computation is also related to the error-algebra framework.

Decoherence-free subspaces and subsystems have already been realized in many important laboratory experiments, of which we mention a few. Consider a system exhibiting electromagnetically induced transparency [16], such as a tenuous vapor of 3-level atoms in the $\Lambda$ configuration (e.g. Strontium), contained in an optical resonator, as described in 17]. Decoherence in this system arises from spontaneous emission, which corresponds to a transition from the excited state to any of the lower lying states. Since spontaneous emission occurs only from the excited state, those states in the orthogonal complement are a decoherence-free subspace.

Zanardi and Rasetti [6] showed that decoherence-free subspaces exist in the phenomenologically important spin-boson model of $N$ spins coupled to a bosonic quantum field, under the assumption that the coupling constants are the same for several of the spins. DFSs also arise as a consequence of a collective coupling to the environment in the JaynesCummings Hamiltonian for $N$ identical two-level atoms coupled to a single mode radiation field [7, 18, 19].

In this short note, we consider all possible representations of the error algebra $\mathfrak{s u}_{3}$, and determine how many of these admit decoherence-free subspaces and noiseless subsystems of various sizes. We prove that for quantum mechanical systems of sufficiently high dimension, decoherence-free subspaces exist for almost all representations of the error algebra. Therefore, our results provide further arguments in favor of the error algebra framework.

Consider a quantum mechanical system with a finite-dimensional Hilbert space $\mathcal{H}$, and let $D=\operatorname{dim}(\mathcal{H})$. This class of systems includes all spin systems, and the state space of any quantum computer. Further suppose that our system is an open quantum system, which means that it interacts with another system, called the "environment," and the latter has 
a Hilbert space $\mathcal{E}$. Unitary evolution for the combined system $\mathcal{H} \otimes \mathcal{E}$ implies non-unitary evolution for the reduced density matrix $\rho: \mathcal{H} \rightarrow \mathcal{H}$ obtained by tracing out the degrees of freedom associated to $\mathcal{E}$.

The interaction Hamiltonian $H: \mathcal{H} \otimes \mathcal{E} \rightarrow \mathcal{H} \otimes \mathcal{E}$ which describes the system-environment coupling necessarily takes the form

$$
H=\sum_{a=1}^{k} F_{a} \otimes B_{a}
$$

where $\left\{F_{a}: a=1 \ldots k\right\}$ is a set of Hermitian operators on $\mathcal{H}$.

If the commutators $\left[F_{a}, F_{b}\right]$ are expressible as linear combinations of the $F_{a}$,

$$
\left[F_{a}, F_{b}\right]=i \sum_{c} f_{a b}^{c} F_{c}
$$

then according to a classic result on decoherence [8], a subspace $V \subset \mathcal{H}$ is decoherence-free if and only if $V$ decomposes as a direct sum of singlets under the action of the $F_{a}$ operators, where a singlet is defined to be a 1-dimensional invariant subspace of $V$.

Assuming (2), we may define an abstract Lie algebra $\mathfrak{g}$ with generators $\left\{x_{a}\right\}$, where $a=1 \ldots k$ and relations $\left[x_{a}, x_{b}\right]=i f_{a b}{ }^{c} x_{c}$. There is then a unique linear map

$$
\phi_{F}: \mathfrak{g} \rightarrow \operatorname{End}(\mathcal{H})
$$

with $\phi_{F}\left(x_{a}\right)=F_{a}$. The map $\phi_{F}$ is a unitary representation of the Lie algebra, since the $F_{a}$ are Hermitian by construction. The reason we make a point to distinguish the abstract Lie algebra $\mathfrak{g}$ from its generators in a particular representation is that we wish to consider the effect of changing the representation without changing the symmetry algebra $\mathfrak{g}$.

For some dimension-dependent number $p$ between 0 and 100, decoherence-free subspaces exist in exactly $p$ percent of all $D$-dimensional open quantum systems with errors acting in all possible ways. In order to allow concrete calculations, we restrict attention to the error algebra $\mathfrak{g}=\mathfrak{s u}_{3}$; the calculations for other Lie algebras differ only in technical aspects.

If $p<5 \%$, one might be tempted to question the utility of the error-algebra framework, since representations which satisfy the necessary criteria would seem to be sparse in the set of all representations. On the other hand, if $p$ is close to $100 \%$, then reliable methods for protecting quantum information from $\mathfrak{s u}(3)$-generated errors are plentiful. Therefore, the precise value of $p$ is of fundamental importance. In this paper, we show that a reasonable 
answer for $D$ on the order of $10^{1} \sim 10^{2}$ is $p \approx 70-90$ percent, and $p$ increases with $D$. Also, $p$ seems to asymptotically approach $100 \%$, so in higher dimensional systems there are many more representations which protect quantum information (provided that the error algebra does not also increase in dimension).

Our results imply that in most dimensions, decoherence-free subspaces exist for almost all representations of the error algebra. However, there are certain dimensions to watch out for: in $D=6$, only three of eight total representations contain a singlet, or 37.5 percent. Therefore, it's especially difficult to preserve quantum information in a 6-dimensional Hilbert space, when the errors are generated by $\mathfrak{s u}_{3}$. Interestingly, this is related to the fact that 3 divides 6 .

While our results are mathematically rigorous, any connection of these results to laboratory studies of decoherence must be classified as highly speculative, for reasons which we now explain. A finite-dimensional open quantum system is specified by a Hilbert space $\mathcal{H}$, system Hamiltonian $H_{s} \in \operatorname{End}(\mathcal{H})$, error algebra $\mathfrak{g}$, and a representation $\phi: \mathfrak{g} \rightarrow \operatorname{End}(\mathcal{H})$. This paper merely determines (for fixed $\mathcal{H}, H_{s}, \mathfrak{g}$ ) the number of representations $\phi$ that support protection of quantum coherence, and the statistical distribution of the sizes of noiseless subsystems over the space of possible representations of the error algebra. We do not discuss the probability that any particular physical system evolves according to the dynamics specified by such a representation.

As a thought experiment, consider an initially isolated $D$-level quantum system, which is then brought into contact with an environment in a controlled way, so that (we suppose) the error algebra is known to be $\mathfrak{s u}_{3}$. One would like to know the true likelihood of encountering a decoherence-free subspace in this situation. The numbers reported in Fig. 2 would answer this question if, by some unknown mechanism, Nature selects one of the available $\mathfrak{s u}_{3^{-}}$ representations at random, with a uniform weighting. As no mechanism which operates this way is currently known, it seems impossible at present to connect Fig. 2 with experiment.

On the other hand, our results show where to look in order to find examples with noiseless subsystems, and also highlight situations in which DF subspaces or noiseless subsystems are rare. As such, these results should help theorists to construct models in which quantum coherence is protected. 


\section{AN EXACT FORMULA FOR THE NUMBER OF SU(3) REPRESENTATIONS IN A FIXED DIMENSION}

\section{A. The Exact Formula}

Let $\xi(n)$ equal the number of irreducible representations of the Lie algebra $\mathfrak{s u}(3)$ in dimension $n$. We compute an explicit formula for this function in Appendix \$

We now fix a dimension $D$ and determine certain facts about the structure of all possible representations (including reducible ones) in that dimension. In particular, we determine the fraction which contain a singlet. Any representation in dimension $D$ may be decomposed into irreducibles, and the dimensions $p_{i}$ of those irreducible components determine a partition 20] of the integer $D$. We say that the representation has shape corresponding to a given partition if the dimensions of its irreducible components are precisely the integers appearing in that partition.

For a partition $\mathfrak{p}=\left(p_{1}, \ldots, p_{n}\right)$ of $D$, a naive guess for the number of representations with shape $\mathfrak{p}$ would be $\prod_{i=1}^{n} \xi\left(p_{i}\right)$. This guess is correct if and only if $\mathfrak{p}$ does not contain repetitions. For example, if $p_{1}=p_{2}$ and $\xi\left(p_{1}\right)>1$ then we are over-counting. In order to properly count these cases, we use a standard combinatorial function, which we now explain.

If $p_{i}$ is repeated $n_{i}$ times in a given partition, we think of the $k=\xi\left(p_{i}\right)$ different $p_{i}$ dimensional irreps as "letters" in an alphabet. The set of distinct $n_{i}$-fold direct sums of these irreps is in 1-1 correspondence with the set of length $n_{i}$ words in $k$ letters, where order of letters in a word is not important.

Lemma 1. Let $S(n, k)$ equal the number of length $n$ strings from an alphabet of $k$ letters, with order not important. Then

$$
S(n, k)=\left(\begin{array}{c}
n+k-1 \\
n
\end{array}\right) .
$$

The recursion relation $S(n, k)=\sum_{\ell=0}^{n} S(n-\ell, k-1)$ follows immediately, and gives an efficient way of calculating the values of $S$. We now return to our objective of counting the total number of $\mathfrak{s u}(3)$ modules in dimension $D$.

Let $\mathcal{P}(D)$ denote the set of all partitions of $D$. Given a set $H$ of non-negative integers, let $\mathcal{P}(H, n)$ denote the set of partitions $n=\sum_{i} p_{i}$ with $p_{i} \in H$ for all $i$, so that $\mathcal{P}(H, n) \subset \mathcal{P}(n)$ 
for all $H \subset \mathbb{N}$. Let $R_{3}$ be the set of possible dimensions of an $\mathfrak{s u}_{3}$-module,

$$
\begin{aligned}
R_{3} & =\{d \in \mathbb{N}: \xi(d) \neq 0\} \\
& =\{1,3,6,8,10,15,21, \ldots\} .
\end{aligned}
$$

Therefore computation of $R_{3}$ reduces to computation of $\xi$, which is done in the appendix.

Theorem 1. Let $\operatorname{Mod}\left(\mathfrak{s u}_{3}, D\right)$ denote the total number of $\mathfrak{s u}(3)$ modules in dimension $D$. Assume that each partition $\mathfrak{p}$ of $D$ is to be expressed in the form $D=\sum_{i=1}^{n} n_{i} p_{i}$ where the $p_{i}$ are all distinct. Then

$$
\operatorname{Mod}\left(\mathfrak{s u}_{3}, D\right)=\sum_{\mathfrak{p} \in \mathcal{P}\left(R_{3}, D\right)} \prod_{i=1}^{n}\left(\begin{array}{c}
n_{i}+\xi\left(p_{i}\right)-1 \\
n_{i}
\end{array}\right)
$$

When $\xi \neq 0$, its most likely value is $\xi=2$, and then the binomial coefficient simplifies to $(n+1$ choose $n)=n+1$. In that case, the partitions that contribute the most are those which maximize the product $\prod_{i=1}^{n}\left(n_{i}+1\right)$, which is the same as maximizing $\prod n_{i}$. Therefore, the largest terms in the sum (5) are those that do not contain singlets; however, the terms which do contain singlets are more numerous. The competition between these two types of terms determines the fraction of representations which contain a singlet, which we analyze in the next section.

\section{B. An algorithm for efficient computation}

The sum in (5) is over $\mathcal{P}\left(R_{3}, D\right)$, the set of partitions of $D$ with parts in $R_{3}$, a specific subset of the positive integers. For efficient computation of (15), it is essential to have an algorithm which lists only the partitions we are interested in, without having to first list

all partitions and then filter out those which do not meet our criteria. As the number of partitions grows exponentially according to the Hardy-Ramanujan formula, any reduction in the number of terms is crucial to make computation of $\operatorname{Mod}\left(\mathfrak{s u}_{3}, n\right)$ even possible.

Fix a set of non-negative integers,

$$
H=\left\{1, n_{1}, n_{2}, \ldots\right\} \subset \mathbb{N}
$$

with $n_{i}<n_{j}$ if $i<j$ and $n_{1}>1$. We present a simple algorithm for explicitly computing the set of partitions of $n$ whose parts lie in $H$. 
A partition $n=\sum_{i=1}^{m} k_{i}$ is said to be in reverse lexicographic order if $k_{1} \geq k_{2} \geq \ldots \geq k_{m}$. We will refer to a partition which is in reverse lexicographic order as an ordered partition for brevity.

Let $P(n, k)$ compute all ordered partitions of $n$ that begin with a number between 1 and $k$. Let

$$
P(n)=\bigcup_{k \geq 0} P(n, k)
$$

denote all ordered partitions. Let

$$
P_{H}(n, k)=\left\{\left(k_{1}, \ldots, k_{m}\right) \in P(n, k) \mid k_{i} \in H \forall i\right\}
$$

denote the subset consisting of those ordered partitions whose elements come from $H$. Similarly define $P_{H}(n)$.

If $\kappa=\left\{k_{1}, \ldots, k_{p}\right\}$ is an ordered partition of an integer $k$, and $\ell>0$ is a positive integer, let $\ell \vee \kappa$ denote the ordered partition of $k+\ell$ given by prepending $\ell$ to $\kappa$, i.e.

$$
\ell \vee \kappa=\left\{\ell, k_{1}, \ldots, k_{p}\right\}
$$

We extend this notation to sets of partitions in the obvious way, so that $\ell \vee P(n)$ denotes $\{\ell \vee \kappa: \kappa \in P(n)\}$.

Theorem 2. The set of partitions whose parts come from $H$ is given by

$$
P_{H}(n)=\bigcup_{k=2}^{n-1}\left\{\begin{array}{cc}
(n-k) \vee P_{H}(k, n-k) & \text { if } n-k \in H \\
\emptyset & \text { otherwise }
\end{array} .\right.
$$

Theorem 2 gives a fast recursive algorithm for enumerating only the partitions of $n$ with parts in $H$. Even on a fast computer, the naive algorithm of enumerating all partitions and subsequently choosing those with the right properties will lead to space problems due to the exponential growth of (6).

\section{Asymptotics for large $n$}

Formula (5) gives a way of evaluating $\operatorname{Mod}\left(\mathfrak{s u}_{3}, n\right)$ numerically, although this is not efficient (or even possible at all for large $n$ ) as it involves a sum over partitions. If $p(n)$ denotes the number of all partitions for $n$, the Hardy-Ramanujan asymptotic formula gives

$$
p(n) \sim \frac{1}{4 n \sqrt{3}} \exp [\pi \sqrt{2 n / 3}]
$$


Good references on this include [20, 21].

Conjecture 1. $\operatorname{Mod}\left(\mathfrak{s u}_{3}, n\right)$ is given, asymptotically for large $n$, by a formula similar to the Hardy-Ramanujan formula (6). Specifically,

$$
\operatorname{Mod}\left(\mathfrak{s u} u_{3}, n\right) \sim \frac{a}{n} \exp \left(b n^{c}\right)
$$

for some positive, real constants $a, b, c$.

The inspiration for this conjecture was Meinardus' theorem (see [20] and references therein), which states roughly that a large class of partition functions have exponential behavior which generalizes that of the Hardy-Ramanujan function.

Nonlinear regression over the interval $[1,110]$ determined that

$$
(a, b, c) \sim(0.0771591,2.70605,0.459802)
$$

which is surprisingly close to the values $0.14,2.57$, and 0.5 given by (6). Fig. 1] shows the curve $(a / n) \exp \left(b n^{c}\right)$ with the approximate values (17), together with points representing the exact values of $\operatorname{Mod}(\mathfrak{s u}(3), n)$ computed using Theorems 1 and 2 for $n \equiv 1(\bmod 3)$.

\section{STATISTICS OF THE SET OF ALL REPRESENTATIONS}

\section{A. Singlets}

As an example to illustrate formula (5) , consider $D=6$. There are 11 partitions of 6 , only 4 of which correspond to direct sum decompositions of representations of $\mathfrak{s u}_{3}$ :

$$
\begin{array}{ll}
p=\{6\} & 2 \text { representations } \\
p=\{3,3\} & 3 \text { representations } \\
p=\{3,1,1,1\} & 2 \text { representations } \\
p=\{1, \ldots, 1\} & 1 \text { representation }
\end{array}
$$

Therefore in $D=6$, three of eight total representations contain a singlet, or $37.5 \%$. By contrast, for $D=5$, because there are no representations of $\mathfrak{s u}(3)$ in dimensions 2,4 and 5 , only two partitions contribute. These are $5=3+1+1$ and $5=1+\cdots+1$ giving 2 and 1 representations respectively. Thus for $D=5$, all three of the three available representations contain a singlet. 


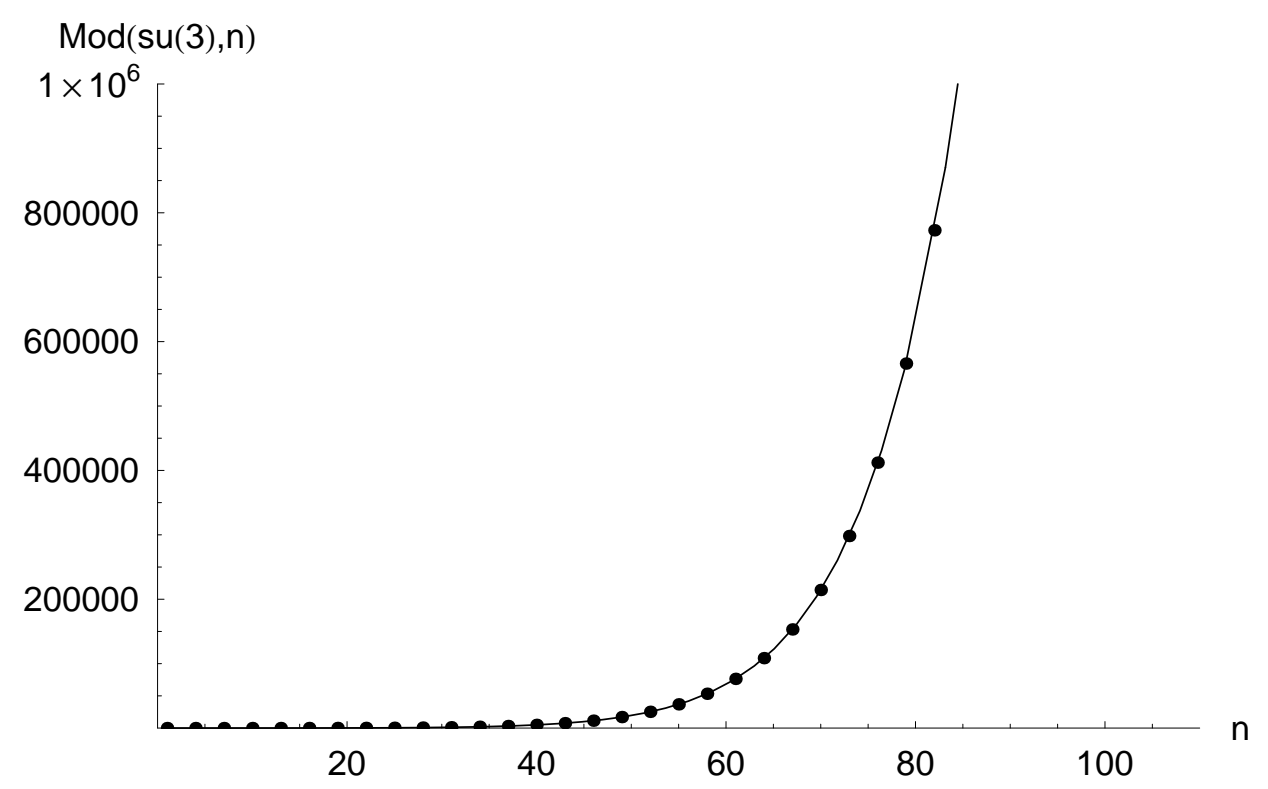

FIG. 1: Graph of $\operatorname{Mod}(\mathfrak{s u}(3), n)$ vs. $n$, with only points $n \equiv 1(\bmod 3)$ shown, fitted by the curve $(a / n) \exp \left(b n^{c}\right)$ with parameter values given by Eqn. (17). The points with $n \equiv 0(\bmod 3)$ could also be fitted to a smooth curve, as could those congruent to $2(\bmod 3)$; those curves have slightly different parameter values.

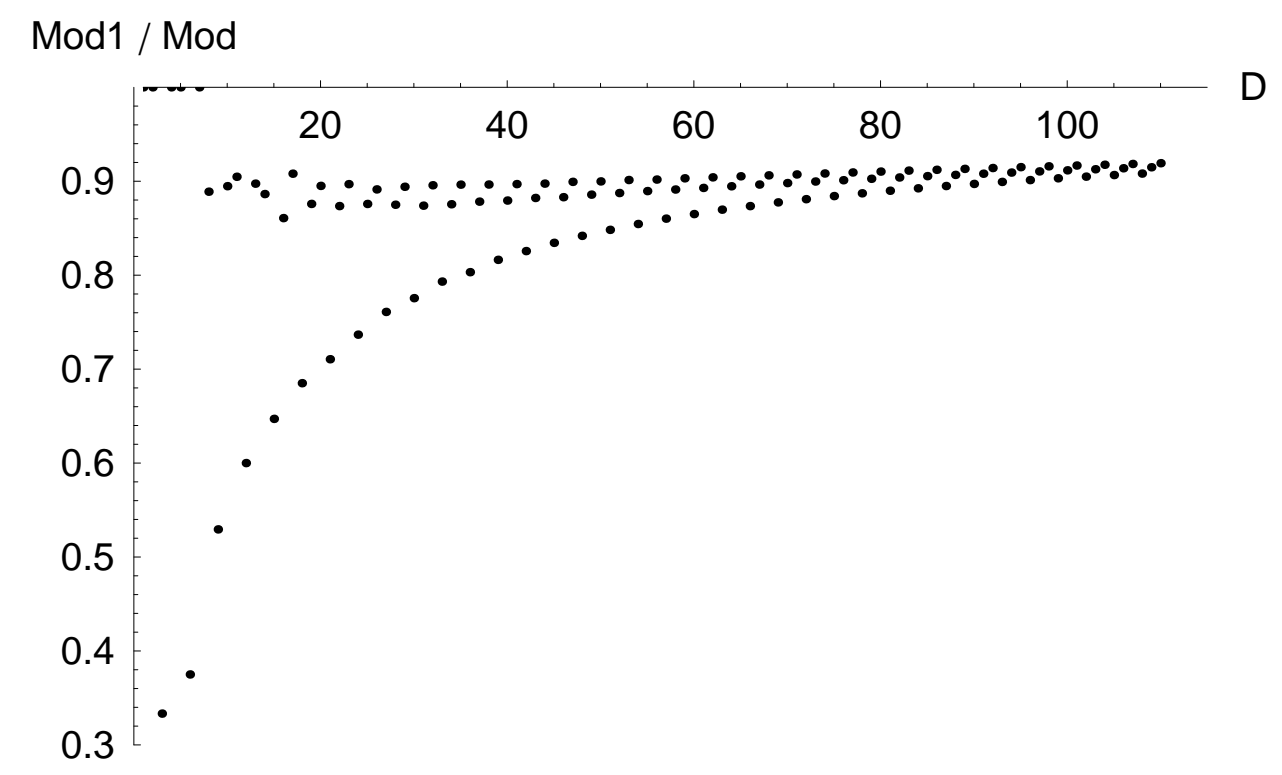

FIG. 2: Fractions of $\mathfrak{s u}(3)$ representations that contain a singlet vs. $D$, for $D \leq 110$. There are three distinct curves corresponding to the distinct congruence classes of $D$ modulo 3 . 
Define $\operatorname{Mod}_{1}\left(\mathfrak{s u}_{3}, D\right)$ by the same formula as (5) but with the sum restricted to partitions containing 1 as one of the elements; the fraction of $\mathfrak{s u}_{3}$ representations that contain a singlet is then $\operatorname{Mod}_{1} / \operatorname{Mod}$. In Fig. 2, we plot $\operatorname{Mod}_{1} / \operatorname{Mod}$ as a function of $D$. The creation of this plot could not have been possible without the computational speedups suggested by Theorems 1 and 2, which is most likely why it has never appeared in the literature before.

Fig. 2 has many fascinating features, which should be of interest to pure mathematicians as well as to physicists. Beginning around $D=10$, one can notice three series in the plot which seem to converge. These three series correspond respectively to the cases $D \equiv$ $0,1,2(\bmod 3)$.

Within each series, the points are very regular and seem to line themselves up along a smooth curve, which at first seems mysterious. It's possible that an explanation for this behavior may be provided by some generalization of Conjecture 1. If we fix the residue class of $n$ modulo 3 , and then find that

$$
\operatorname{Mod}_{1}\left(\mathfrak{s u}_{3}, n\right) \sim\left(a_{1} / n\right) \exp (g(n))
$$

for some function $g$, then we have

$$
\operatorname{Mod}_{1} / \operatorname{Mod} \sim \frac{a}{a_{1}} \exp \left(b n^{c}-g(n)\right) .
$$

If $g(n)$ is such that $b n^{c}-g(n)$ is negative but approaches zero monotonically from below, then we obtain the behavior observed in Fig. 2.

\section{B. Noiseless Subsystems}

Decoherence-free subspaces do not provide the most general method for decoherence-free encoding of quantum information. Knill, Laflamme, and Viola [10] discovered a method for decoherence-free encoding using subsystems. Zanardi soon thereafter realized that this allowed a unification of many seemingly unrelated ideas for reducing decoherence [13]. Kempe et al. developed a general theory of universal quantum computation based on the decoherence-free (or noiseless) subsystem concept [22].

Irreducible representations of the error algebra do not possess noiseless subsystems. We therefore devote this section to determining how many representations contain noiseless subsystems of various sizes. Our results are presented in Figs. 3 and 4 and the surrounding discussion. 
A representation $\phi$ of $\mathfrak{g}$ lifts to a unique associative algebra homomorphism $\tilde{\phi}$ of the universal enveloping algebra $\mathcal{U}(\mathfrak{g})$, by the universal property most elegantly expressed in the commutative diagram

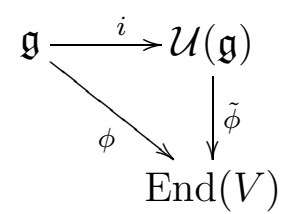

The action of $\tilde{\phi}$ is simply to convert the tensor product to matrix multiplication, i.e. $\tilde{\phi}(x \otimes y)$ $=\phi(x) \cdot \phi(y)$, etc.

Lemma 2. If $\phi$ is an irreducible faithful representation and if $\mathfrak{g}$ is a semisimple Lie algebra, then $\tilde{\phi}$ is surjective.

(The proof of lemma 2 is an easy exercise.)

Each irreducible representation $\phi$ will have the property that $\tilde{\phi}(\mathcal{U}(\mathfrak{g}))$ will be the full matrix algebra $\operatorname{End}(V)$. On the other hand, noiseless subsystems take advantage of a basis in which $\tilde{\phi}(\mathcal{U}(\mathfrak{g}))$ is block diagonal. Therefore, these blocks correspond to a decomposition of the Hilbert space $\mathcal{H}$ into irreducible subrepresentations of the original representation coming from the $F$-operators,

$$
\mathcal{H}=\left(\bigoplus^{n_{1}} V_{d_{1}}\right) \oplus \cdots \oplus\left(\bigoplus^{n_{r}} V_{d_{r}}\right)
$$

where the subspaces are labelled by dimension, $\operatorname{dim}\left(V_{d_{i}}\right)=d_{i}$. In this situation, we can send an $N$-dimensional vector, where $N=\sum_{i} n_{i}$, through the quantum channel unaffected by decoherence.

If the representation of the $F$-operators does indeed have such a decomposition, then it corresponds naturally to a partition

$$
D=\sum_{i=1}^{r} n_{i} d_{i}
$$

One may now compute the percent of all representations in dimension $D$ which preserve an $N$-dimensional vector, for various $N<D$. Recall that $R_{3}$, which was defined in (4), denotes the set of positive integers which are the dimension of an irreducible $\mathfrak{s u}_{3}$ module.

Theorem 3. Let each partition $\mathfrak{p}$ of $D$ be expressed as $D=\sum_{i=1}^{n} n_{i} p_{i}$. The number of representations in dimension $D$ which preserve an $N$-dimensional vector is given by

$$
\sum_{\substack{\mathfrak{p} \in \mathcal{P}\left(R_{3}, D\right) \\
\text { with } N=\sum_{i} n_{i}}} \prod_{i=1}^{n}\left(\begin{array}{c}
n_{i}+\xi\left(p_{i}\right)-1 \\
n_{i}
\end{array}\right),
$$




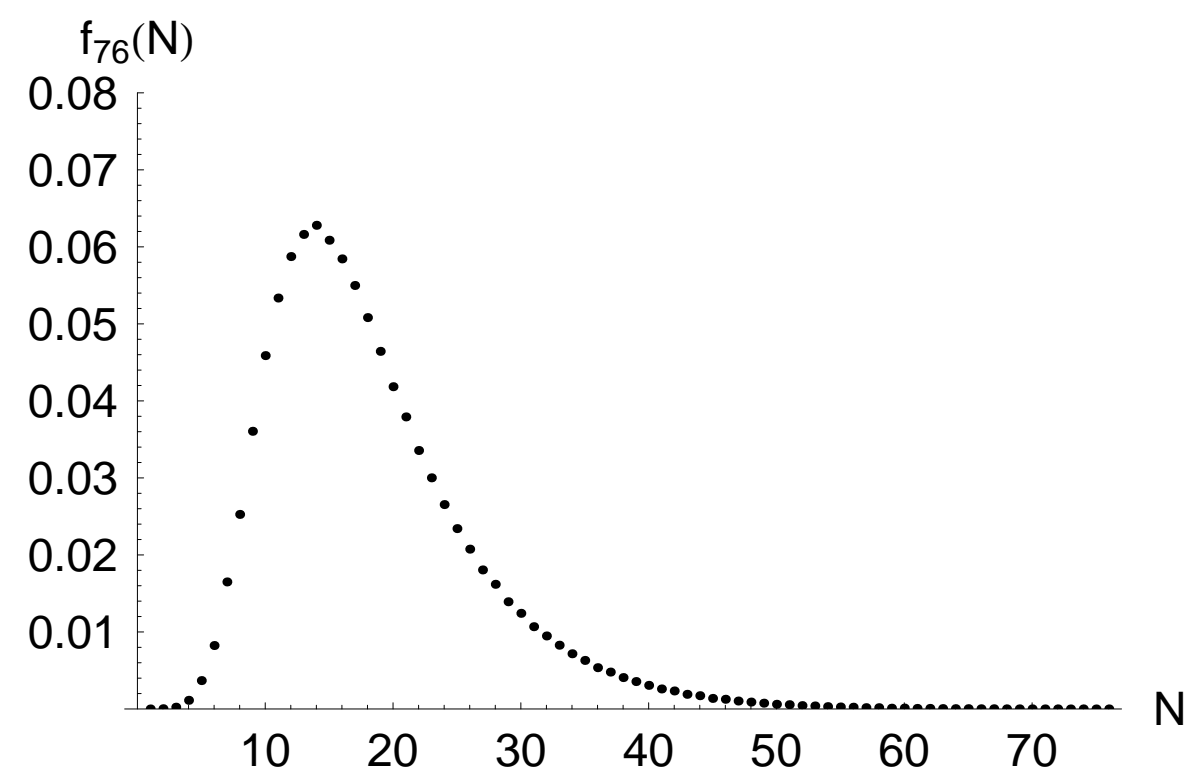

FIG. 3: the fraction $f_{d}(N)$ of all representations in dimension $d=76$ which preserve an $N$ dimensional noiseless subsystem, as a function of $N$. The peak is at $\sim d / 5$.

where the sum is over partitions $\mathfrak{p}$ with $N$ total repetitions, and with each $p_{i} \in R_{3}$.

Like theorem 1, this theorem also gives a method for computing something which would otherwise have seemed elusive. In this case we can plot, for any particular dimension $d$, the fraction $f_{d}(N)$ of all representations in dimension $d$ which preserve an $N$-dimensional noiseless subsystem, as a function of $N$. The results (figs. 3 and 4) are illuminating.

First note from the definition of $f_{d}(n)$ that

$$
\sum_{n=1}^{d} f_{d}(n)=1,
$$

since every representation is counted once. Therefore, we may view $f_{d}(n)$ as the probability distribution function for a random variable. Further, if $d \gg 1$, then the collection of points $\left\{\left(n, f_{d}(n)\right): n=1 \ldots d\right\}$ forms a smooth curve, ${ }^{1}$ which must then subtend unit total area; see figure 3

Figure 4 shows the exact values of $f_{100}(n)$ together with a fit to the standard inverted beta statistical distribution, defined by

$$
f(x)=n_{\alpha, \beta}^{-1} x^{\alpha-1}(1+x)^{-\alpha-\beta}
$$

\footnotetext{
${ }^{1}$ For $\mathfrak{s u}_{3}$, it is sufficient to take $d>50$ to see the smooth shape.
} 


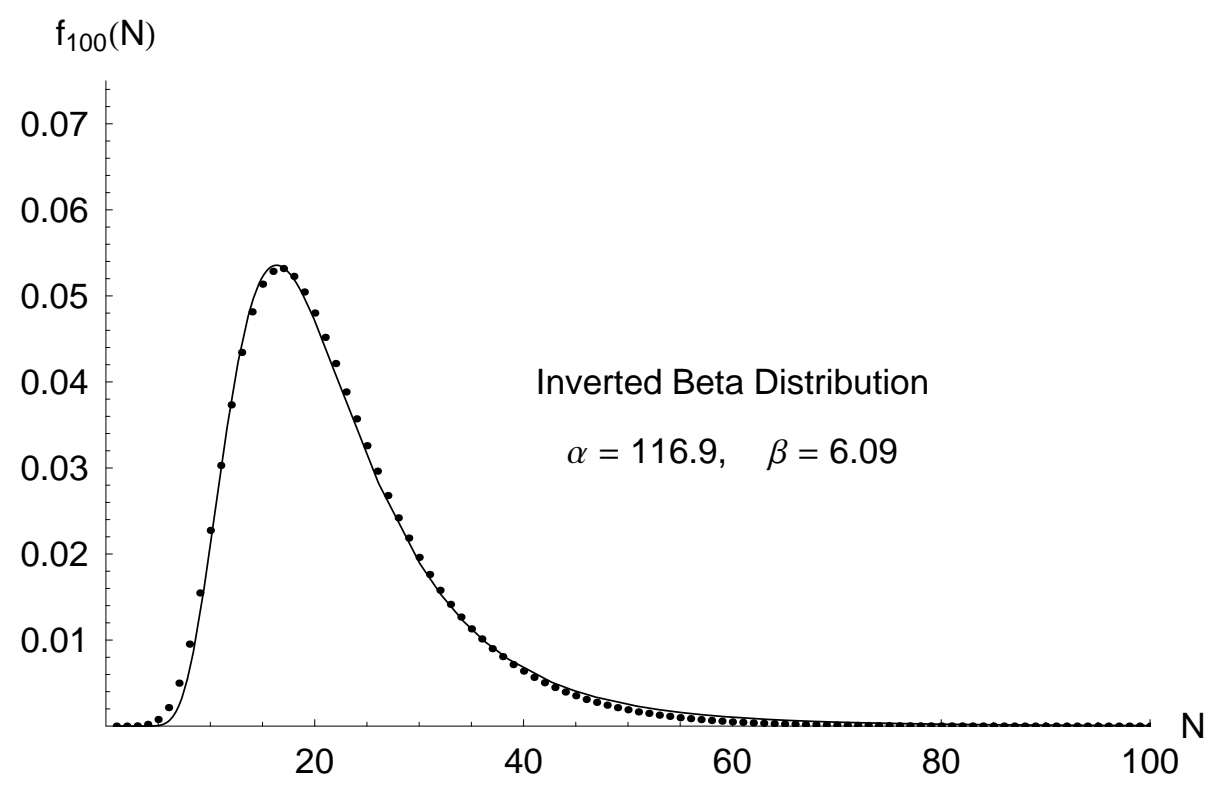

FIG. 4: Data points represent exact computations of $f_{100}(N)$, the fraction of representations in dimension $d=100$ which preserve an $N$-dimensional noiseless subsystem. The points are fitted to an inverted beta distribution (see equation (9)) with $\alpha \sim 116.907$ and $\beta \sim 6.091$. Comparing with figure 3, which has $d=76$, we note that in both cases, the peak is near $d / 5$.

where $\alpha, \beta>0$ and $n_{\alpha, \beta}=\int_{0}^{1} x^{\alpha-1}(1-x)^{\beta-1} d x$ is a normalization constant.

Since the distribution $f_{d}(n)$ is not mathematically known to take the form (9), it is of interest to determine how close the agreement is. It is important that the points in Figure 4 are not data; they are exact values for fractions. Therefore, the relevant "scale" in the plot is the dimensionless number 1 , and a particularly meaningful measure of accuracy is simply $\Delta_{f}:=(1 / d) \sum_{i=1}^{d}\left|y_{i}-f\left(x_{i}\right)\right|$, the average deviation of the model from the "data," measured with respect to the only meaningful "scale," which is 1 . For the example depicted in Figure 4, we compute $\Delta_{f}=5.2 \times 10^{-4}$.

This agreement is remarkable, and seems to improve as $d$ increases; therefore, we come to the unexpected conclusion that $f_{d}(n)$ is asymptotically (for $d \gg 1$ ) equivalent to an inverted beta distribution!

Further, inspection of many examples shows that for sufficiently large $d$, the shape of the function $f_{d}(n)$ does not depend strongly on $d$. More precisely, as $d$ is increased, the function receives overall scale factors for the horizontal and vertical axes. In all observed examples, the curve always has a peak at around $d / 5$. Given that the distribution function $f_{d}(n)$ is 
completely insensitive to the details of the system Hamiltonian $H_{S}$ and to the details of the heat bath to which the system is coupled, and displays a simple scaling behavior with respect to the dimension of the system Hilbert space, one is tempted to term this "universal behavior."

\section{CONCLUSIONS AND OUTLOOK}

As soon has one has appreciated the importance of representations of the $\mathfrak{s u}_{n}$ Lie algebras in physics, natural questions arise. How many of these representations exist, up to equivalence, for fixed $d=\operatorname{dim}(\mathcal{H})$ ? How does the number grow with $d$ ? Suppose that we reduce each representation into irreducibles. What is the statistical distribution function which describes the relative frequencies of the different possible reductions? The present work has given answers (in some cases only partial) to each of these questions, while interpreting the results within the unified understanding of quantum decoherence that has emerged in the last decade [22, 23]. Many unanswered questions remain, such as a proof of Conjecture 1, a better explanation of Fig. 2 than the heuristic one given in Sec. III A analogues of these results for other Lie algebras, and a fundamental derivation of the " $d / 5$ rule," by which we mean the curious fact that the curves plotted in Sec. IIIB seem to always peak around $N=d / 5$.

\section{Acknowledgements}

The author gratefully acknowledges helpful discussions with Gregg Zuckerman and Noam Elkies, and helpful comments by the referee on the first draft.

\section{APPENDIX A: COMPUTING THE NUMBER OF IRREDUCIBLE REPRESEN- TATIONS IN ANY DIMENSION}

In this appendix, we describe how to efficiently compute the function $\xi(n)$ that was used elsewhere in the paper.

For a Young diagram $\lambda$, we let $d(\lambda, \mathfrak{g})$ denote the dimension of the corresponding irreducible module of the Lie algebra $\mathfrak{g}$. Unless noted otherwise, we will henceforth assume 
$\mathfrak{g}=\mathrm{su}_{r}$, and denote the dimension by $d(\lambda, r)$. Further, we often set $r=3$, in which case we will suppress $r$ from the notation. Young diagrams for $\mathrm{su}_{3}$ are characterized by row lengths $n_{1}, n_{2}$ with $n_{1} \geq 0$ and $0 \leq n_{2} \leq n_{1}$. The fundamental representation is $\lambda=(1,0)$ while the adjoint is $\lambda=(2,1)$. The representations $\left(n_{1}, n_{2}\right)$ and $\left(n_{1}, n_{1}-n_{2}\right)$ are conjugate to each other and have the same dimension. Representations of the form $(2 n, n)$ are self-conjugate.

The dimension given by the Weyl character formula 24] is then

$$
\begin{aligned}
d\left(n_{1}, n_{2}\right) & =\frac{1}{2}\left(n_{1}+2\right)\left(n_{2}+1\right)\left(n_{1}-n_{2}+1\right) \\
& =x y(x-y) / 2
\end{aligned}
$$

where $x=n_{1}+2$, and $y=n_{2}+1$.

We now compute the total number $N_{D}$ of irreducible representations of $\mathfrak{s u}_{3}$ with dimension less than $D$, by finding, for each fixed $n_{1}$, the number of $n_{2}$ which satisfy $d\left(n_{1}, n_{2}\right) \leq D$, and then sum over $n_{1}$. Expressing the sum in terms of $x=n_{1}+2$ gives simpler notation.

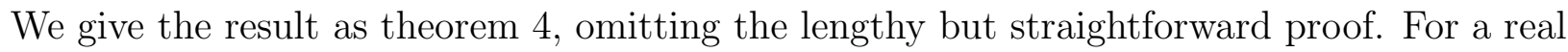
number $\gamma \in \mathbb{R}$, we let $\lfloor\gamma\rfloor$ denote the greatest integer less than $\gamma$. Similarly, $\lceil\gamma\rceil$ denotes the least integer above $\gamma$.

Theorem 4. Let $N_{D}$ denote the total number of irreducible representations of $\mathfrak{s u}(3)$ with dimension less than $D$. Then $N_{D}$ is given exactly by

$$
N_{D}=\frac{1}{2} l_{D}\left(l_{D}-1\right)-\sum_{x=k_{D}+1}^{l_{D}}\left(\left\lfloor y_{+}\right\rfloor-\left\lceil y_{-}\right\rceil+1\right) .
$$

where $k_{D}:=\lfloor 2 \sqrt[3]{D}\rfloor, l_{D}:=\left\lfloor\frac{1}{2}(1+\sqrt{1+8 D})\right\rfloor$, and

$$
y_{ \pm}=\frac{x-2}{2} \pm \frac{1}{2}\left(x^{2}-8 D / x\right)^{1 / 2} .
$$

Corollary 1. The exact number $\xi(D)$ of irreducible representations in dimension $D$ is given by

$$
\xi(D)=N_{D+\frac{1}{2}}-N_{D-\frac{1}{2}} .
$$

For determining the number of irreducible representations of dimension $D$, eqns. (A1)(A2) provide a radical computational speedup over the naive algorithm of enumerating all possible Young diagrams and computing the dimension of each. These equations may be 
implemented with an optimized C program.

[1] C. H. Bennett and P. W. Shor, IEEE Trans. Inform. Theory 44, 2724 (1998), information theory: 1948-1998.

[2] D. Gottesman, Phys. Rev. A (3) 54, 1862 (1996).

[3] E. Knill and R. Laflamme, Phys. Rev. A (3) 55, 900 (1997).

[4] A. R. Calderbank, E. M. Rains, P. W. Shor, and N. J. A. Sloane, Phys. Rev. Lett. 78, 405 (1997).

[5] P. Zanardi and M. Rasetti, Modern Phys. Lett. B 11, 1085 (1997).

[6] P. Zanardi and M. Rasetti, Phys. Rev. Lett. 79, 3306 (1997), quant-ph/9705044.

[7] P. Zanardi, Phys. Rev. A57, 3276 (1998), quant-ph/9705045.

[8] D. A. Lidar, I. L. Chuang, and K. B. Whaley, Phys. Rev. Lett. 81, 2594 (1998), quant$\mathrm{ph} / 9807004$.

[9] D. A. Lidar, D. Bacon, and K. B. Whaley, Phys. Rev. Lett. 82, 4556 (1999), quant-ph/9809081.

[10] E. Knill, R. Laflamme, and L. Viola, Phys. Rev. Lett. 84, 2525 (2000).

[11] L. Viola and S. Lloyd, Phys. Rev. A (3) 58, 2733 (1998).

[12] M. H. Freedman, A. Kitaev, M. J. Larsen, and Z. Wang, Bull. Amer. Math. Soc. (N.S.) 40, 31 (2003), mathematical challenges of the 21st century (Los Angeles, CA, 2000).

[13] P. Zanardi, Phys. Rev. A (3) 63, 012301, 4 (2001).

[14] M. Rasetti and P. Zanardi, in Proceedings of the Adriatico Conference on Quantum Interferometry, III (Trieste, 1999) (2000), vol. 48, pp. 559-572.

[15] P. Zanardi and S. Lloyd, Phys. Rev. Lett. 90, 067902 (2003), quant-ph/0208132.

[16] S. Harris, Physics Today 50, 36 (1997).

[17] D. A. Lidar and K. B. Whaley, in Irreversible Quantum Dynamics, edited by F. Benatti and R. Floreanini (Springer-Verlag, Berlin, 2003), vol. 622 of Lecture Notes in Physics.

[18] L.-M. Duan and G.-C. Guo, Phys. Rev. A (3) 57, 2399 (1998).

[19] L.-M. Duan and G.-C. Guo, Phys. Rev. A (3) 58, 3491 (1998).

[20] G. E. Andrews, The theory of partitions, Cambridge Mathematical Library (Cambridge University Press, Cambridge, 1998), reprint of the 1976 original.

[21] G. H. Hardy and E. M. Wright, An introduction to the theory of numbers (The Clarendon 
Press Oxford University Press, New York, 1979), 5th ed.

[22] J. Kempe, D. Bacon, D. Lidar, and K. Whaley, Phys. Rev. A (3) 63, 042307 (2001).

[23] W. G. Ritter, J. Math. Phys. 46, 082103 (2005).

[24] W. Fulton and J. Harris, Representation theory, vol. 129 of Graduate Texts in Mathematics (Springer-Verlag, New York, 1991), a first course, Readings in Mathematics. 